\title{
Herramienta de software para el pronóstico de demanda horaria de potencia eléctrica en el sistema eléctrico de codensa S.A. ESP
}

\author{
Software package to diagnose the hourly demand for electricity in \\ the electric system ofcodensa S.A. ESP
}

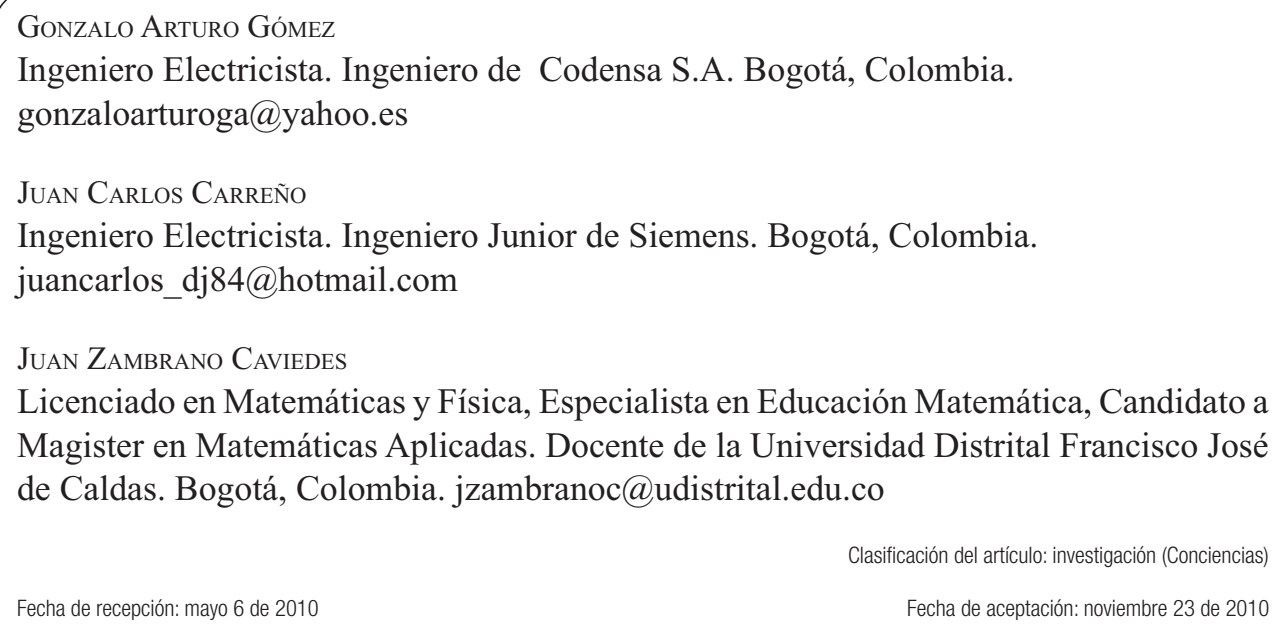

Palabras clave: Calidad de potencia, Demanda de energía, Predicción, Series de tiempo..

Key words: Power quality, Demand for energy, Forecast, Time Series.

\section{RESUMEN}

El país en busca de mejorar y optimizar los recursos energéticos del sistema eléctrico de potencia presenta en su marco regulatorio la necesidad de que todos los operadores de red del país entreguen un pronóstico de energía y potencia horaria. En este artículo se describe un modelo dinámico para el pronóstico de demanda horaria a corto plazo, desarrollando un soporte lógico, con el fin de obtener una reducción del error aproximadamente del $2 \%$, con respecto al modelo actual utilizado por Codensa S.A. para pronóstico de demanda.

\section{Abstract \\ In pursuit of improving and optimizing the energetic resources of its electric power system, the coun- try, in its regulatory framework, has set the need of diagnosing hourly energy and power demand.}




\section{con-ciencias}

This paper presents a dynamic model to forecast the hourly demand in the short term, and logical support will be developed in order to obtain an approximate $2 \%$ error reduction in comparison with CODENSA's present model to diagnose demand.

\section{Introducción}

El sistema eléctrico de potencia del país, siendo un mercado eléctrico desregularizado, presenta una serie de marcos regulatorios que exige a todos los operadores de red del país un pronóstico de energía y potencia horaria, el cual debe ser enviado semanalmente al Centro Nacional de Despacho (CND) con el fin de que éste pueda realizar la operación y programación de las unidades de generación que diariamente salen seleccionadas en el despacho económico, con el propósito de que no se tengan que realizar nuevas programaciones con unidades de generación más costosas para atender la demanda o, en el caso contrario, prescindir de generaciones programadas, todo ello implicando mayores costos por generación.

Codensa S.A. ESP, como empresa distribuidora de energía eléctrica, semana tras semana tiene la obligación de enviar al CND un pronóstico de demanda de energía diaria, por esta razón en este proyecto se desarrolla una herramienta de software para pronóstico de demanda horaria a corto plazo del sistema eléctrico de Codensa S.A., a partir de técnicas matemáticas deductivas y técnicas de inteligencia artificial.

Dentro del alcance del proyecto también se estudia el estado del arte de las metodologías y los modelos de predicción de demanda horaria y se revisan los soportes lógicos manejados en la actualidad para dicho fin.

\section{Antecedentes y estado actual}

En la actualidad, el marco regulatorio del mercado eléctrico colombiano ha tenido mayor interés en satisfacer la demanda energética de todos los usuarios con unos indicadores de calidad, seguridad y confiabilidad. Esto en parte, al actual mercado liberalizado del país y al auge mundial en optimizar los recursos energéticos lo que conlleva al uso racional de energía.

El sistema eléctrico de potencia requiere adaptarse a la demanda solicitada, ya que es necesario mantener un servicio continuo del suministro. Sin embargo, los sistemas eléctricos no son sistemas adaptativos, por esta razón se requiere contar con un estimado del valor demandado por dicho sistema para poder realizar las modificaciones y cumplir con el servicio.

Cada día la realización de un muy acertado pronóstico de energía y de potencia eléctrica se convierte en un factor relevante e importante debido a la necesidad de poder realizar la operación y programación de las unidades de generación que diariamente salen seleccionadas en el despacho económico. Por otro lado, este factor se ha convertido en una misión de los diferentes operadores de red, los cuales desarrollan una planeación operativa y comercial a partir de este cálculo.

Al disminuir las desviaciones del pronóstico se reduce el riesgo de realizar redespachos donde se deban usar unidades de generación más costosas o, por el contrario, se deba prescindir de generaciones programadas anteriormente. Es por ello que en este documento se analiza y se realiza un modelo para el pronóstico de demanda horaria a corto plazo, para permitir así, a través de este cálculo, que los OR (Operador de Red) tomen decisiones que puedan prevenir las sobrecargas y que al mismo tiempo se puedan evitar ciertos eventos, como son las fallas de los equipos o apagones que implican grandes 


\section{con-ciencias}

pérdidas; de esta manera, se proporciona una mejor confiabilidad del sistema eléctrico.

En cuanto al marco regulatorio, la resolución CREG (Comisión de Regulación de Energía y Gas) 097 de 2008 establece un esquema de compensaciones cuando ocurren eventos en el STR(Sistema de Transmisión Regional) por ENS (Energía NO Suministrada), teniendo en cuenta el porcentaje que esta energía representa frente al pronóstico, colocando topes de $2 \%$. Esto implicaría que el error de pronóstico será definitivo para determinar si a nivel horario se supera el límite de $2 \%$. Por lo tanto, los operadores de red se ven en la necesidad de buscar alternativas de herramientas o modelos que disminuyan las desviaciones del pronóstico y mejoren la calidad del mismo [1].

Existen diferentes técnicas de predicción de sistemas, las cuales se dividen en tres áreas: técnicas deductivas matemáticas, técnicas estadísticas y técnicas mediante inteligencia artificial. En la actualidad, en Colombia y en muchos países del mundo utilizan diferentes métodos en relación al pronóstico de demanda de energía, los más utilizados son modelos ARIMA, ARMAX, Regresiones Lineales, Promedios Móviles y se han realizado algunos estudios con Redes Neuronales e Inteligencia Artificial.

A continuación se presenta el estado del arte de metodologías y soportes lógicos utilizados para la predicción de demanda horaria en Colombia, España y México.

\subsection{Estado actual en Colombia}

En Colombia los OR son los encargados de entregar semanalmente al ente regulatorio CREG y al CND la estimación de demanda de energía que sus clientes consumirán en los siguientes siete días. Para esto, cada uno ha implementado un modelo para su estimación a partir del histórico de demanda horaria de su sistema.
Tenemos el caso de EPSA (Empresa del Pacifico S.A.) el cual actualmente utiliza un modelo de promedios móviles para el pronóstico horario de energía, éste está programado en una aplicación de EXCEL. Este modelo se estudia en detalle en Modelo de promedios móviles para el pronóstico horario de potencia y energía eléctrica [2].Uno de los aportes más relevantes de esta investigación en nuestro modelo fue la conclusión de la estrecha relación e importancia que existe entre los datos más recientes con el dato a pronosticar.

En el caso de la Empresa de Energía de Pereira (EEP) se utilizó un modelo $\operatorname{ARIMA}(1,0,0)(2,1,0)$ para su pronóstico horario de demanda, el cual se desarrolló bajo el paquete estadístico SPSS (Statistical package for the social sciences) y se implementó en una aplicación de Excel. Este modelo se estudia en [3]. De este trabajo se destaca la forma en la que se analizó la serie de tiempo, explicando cada uno de los componentes de la misma.

Todos los OR del país han adoptado un método para su respectivo pronóstico de demanda horaria, XM Compañía de Expertos en Mercados S.A.E.S.P., encargada de prestar los servicios de planeación y coordinación de la operación de los recursos del SIN (Sistema Interconectado Nacional), ha realizado un seguimiento a través de indicadores de demanda de las UCP's (Unidades de Control de Pronóstico), término que acordó la UPME (Unidad de Planeación Minero Energética) para el estudio y análisis de proyección de demanda.

La estructura y clasificación de las UCP's se presenta en [4]. La referencia [5] muestra los histogramas de los pronósticos de cada OR del sector eléctrico colombiano y realiza una evaluación de cada uno, a continuación tenemos la valoración efectuada.

Los indicadores están suministrados en promedio mes por cada UCP's y están dados por:

- Pronóstico de buena calidad entre el $\pm 2 \%$.

- Pronóstico de mala calidad superior de $\pm 4 \%$. 


\section{con-ciencias}

A manera de discriminar esta valoración por OR y no por UCP's hicimos el siguiente análisis:

Dentro de los pronósticos con oportunidad de mejoramiento se encuentran: Codensa, Cundinamarca, Ebsa, Enelar, Cens, Essa, Epsa, Tulua, Cartago, Electrocosta, Electrocaribe, Emcali, Quindio, con un margen que se encuentra entre el $\pm 2.5 \%$ y $\pm 5 \%$.

Dentro de los pronósticos con indicadores de mala calidad se encuentran: EPM, Etaservicios, Dispac, Cedelca, Electrohuila, Emsa y Energuaviare, con un margen que se encuentra entre el $\pm 3 \%$ y el $\pm 8 \%$. Con el fin de mitigar el error a la hora de hacer el pronóstico de demanda de energía eléctrica, XM mantiene un control intensivo por mes, revisando las series de tiempo de cada UCP's y sometiéndolas a controles estadísticos, a las que también se les hacen seguimientos de control y de calidad por parte del Comité de Distribución [4].

Para la estimación del pronóstico de demanda horaria XM, quien tiene a cargo la operación y mantenimiento del CND utiliza un modelo ARIMA del cual no se pudo obtener sus características por ser información confidencial. En cuanto al modelo actual utilizado por Codensa S.A. ESP, objeto de estudio en este proyecto, se tiene una aplicación en Excel que utiliza los modelos de regresión lineal, exponencial y media móvil para pronosticar la demanda de energía horaria; a partir de este cálculo y con la experiencia del personal que realiza esta labor se determina la estimación de demanda.

\subsection{Estado actual en otros países}

Dentro de la investigación realizada se encontró que España utiliza un modelo Box-Jenkins para el pronóstico de demanda de energía a corto plazo. Entre las diferencias encontradas con los modelos utilizados en nuestro país, se encuentra la modelización de las variables meteorológicas y la vinculación al modelo de una variable de laboralidad, este modelo se describe en [6].
Una de las características más relevantes que se encontró es el soporte lógico que utilizan para el cálculo del pronóstico, la aplicación corre bajo la misma plataforma del SCADA del Centro de Control Eléctrico Español (CECOEL);cuando la demanda real no sigue a la prevista, ejecuta una nueva predicción con el modelo. Siendo la producción la que no se ajusta al consumo, modifica la generación recurriendo a los mecanismos que, con diferentes horizontes temporales, posee el sistema para asegurar el necesario cumplimiento de la relación generación igual a demanda. Aunque en el mercado colombiano no aplicaría este modelo para las UCP's, debido a que se tiene una diferencia de siete días, se debería implementar este control dinámico en el CND para el control conjunto de unidades.

Uno de los aportes más destacados de esta investigación en nuestro modelo fue la idea de utilizar una serie de filtros dependiendo del pronóstico que se pretendía calcular, lo que más adelante llamaremos filtros dinámicos.

En cuanto al modelo utilizado en México para realizar el pronóstico de demanda horaria a corto plazo se encontró que en el área peninsular de la República Mexicana utilizan un modelo de regresión lineal, basado en una clasificación de aspectos que denominaron factores comunes, factores ocasionales y factores estacionales, como se muestra en [7].

El modelo está dado por la ecuación (1):

$$
Y_{t}=B_{0}+\sum_{i=1}^{p} B_{i} X_{t i}+\varepsilon_{t}
$$

Donde:

$Y_{t}=$ Demanda pronosticada en el periodo t.

$X_{t i}=$ Factores que influyen en la demanda.

$\varepsilon_{t}=$ Todos los factores que no consideran las variables independientes. 


\section{con-ciencias}

$B_{0}=$ Parámetros desconocidos de las variables dependientes

La aplicación utilizada para el desarrollo de este modelo es un paquete llamado Forescast Pro XE, el cual tiene incorporado una serie de aplicaciones estadísticas que complementa el cálculo de la estimación de demanda.

\section{Metodología}

Antes de considerar la metodología para el pronóstico de demanda horaria a corto plazo utilizada en este proyecto, se mostrarán algunos aspectos importantes que se tuvieron en cuenta antes del desarrollo del modelo.

\subsection{Análisis de la serie de tiempo}

Para el desarrollo de este proyecto se contó con el histórico de demanda del sistema eléctrico que maneja el OR Codensa S.A. ESP, el cual tenía almacenados registros hora-hora desde el 2004. El análisis de la serie de tiempo comenzó con la búsqueda de valores atípicos de demanda que fueron normalizados con la media de los días adyacentes al valor erróneo encontrado.

Dentro de esta normalización se encontró que los mayores errores se habían dado en el periodo 2004 y 2005; al realizar un diagnóstico a la base de datos se concluyó que el problema se había originado en el ingreso de la información. Con este proceso se garantizó que la serie de tiempo de demanda horaria se encontraba con todos los datos válidos, evitando así errores futuros en el pronóstico.

Luego de la normalización de los datos, se empezó a estudiar y a analizar el comportamiento de la serie de tiempo con ayuda del paquete estadístico Eviews en versión demo. Se inició con unas pruebas realizadas a la serie de tiempo, en la que se incluyó la prueba de estacionalidad y raíz unitaria, además se graficaron sus correlogramas en busca de relaciones de datos dentro de la misma serie.

\subsection{Tipificación}

Durante el desarrollo del proyecto se vio la necesidad de realizar una tipificación de la serie de tiempo, debido a que dentro del análisis de la misma se encontraron algunas relaciones que era necesario clasificar para un mejor procesamiento de la información. En los siguientes apartados se presentan y se argumentan las características de esta tipificación.

\subsubsection{Comportamientos típicos}

A partir del histórico de demanda de energía eléctrica, se graficaron todos los días de una semana hora a hora y se observó claramente que todos los días de la semana no presentaban el mismo comportamiento, debido a esto se procedió a tomar otras semanas para verificar si todos los días presentaban las mismas características. Este análisis arrojó como resultado que la forma de la curva de demanda era similar y se concluyó que para una semana normal sin días festivos existía una relación que conlleva a clasificar la semana en tres grupos diferentes como se muestra en la Tabla 1.

Tabla 1. Clasificación de días de una semana normal de acuerdo al comportamiento de la demanda.

\begin{tabular}{|c|c|c|}
\hline \multicolumn{3}{|c|}{ GRUPO } \\
\cline { 1 - 1 } $\mathbf{1}$ & $\mathbf{2}$ & $\mathbf{3}$ \\
\cline { 1 - 1 } LUNES & & \\
\cline { 1 - 1 } MARTES & & \multirow{2}{*}{ SÁBADO } \\
\cline { 1 - 1 } MIÉRCOLES DOMGO \\
\cline { 1 - 1 } JUEVES & & \\
\cline { 1 - 1 } VIERNES & & \\
\hline
\end{tabular}




\section{con-ciencias}

Esta conclusión se puede apreciar claramente en la Figura 1, la cual muestra cómo es el comportamiento de la demanda en una semana típica sin días festivos.

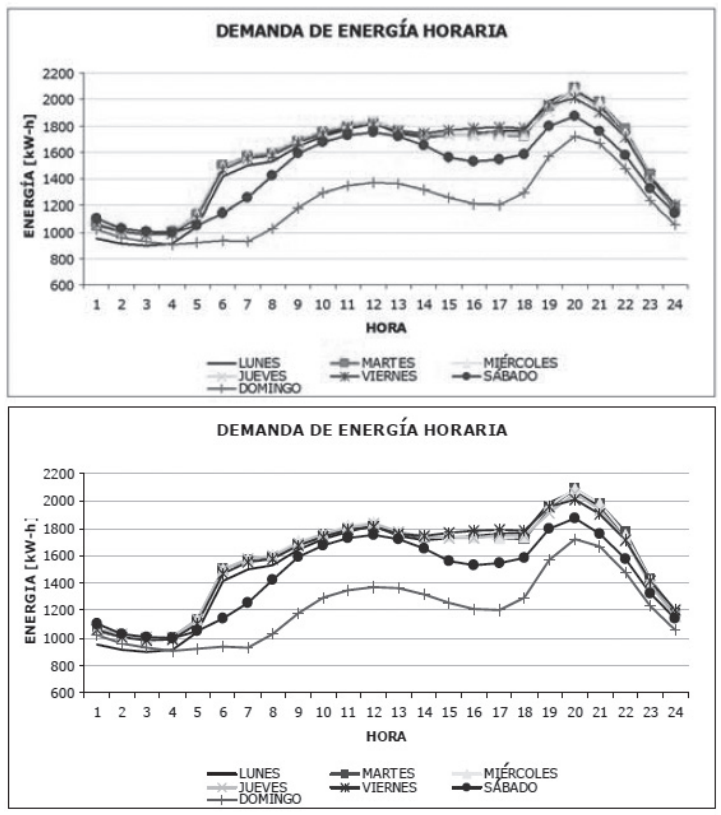

Figura 1. Demanda de energía horaria de una semana típica.

Como se muestra en la Figura 1, los días lunes, martes, miércoles, jueves y viernes presentan un comportamiento bastante similar, mientras que el sábado y el domingo su forma varía en gran proporción, esta variación es más pronunciada entre las horas 4 y 22.

En algunos casos el comportamiento de la demanda varía en un día específico, como sucede con los días festivos, esto se puede apreciar en la Figura 2, la cual muestra que algunos días lunes festivo tienen un comportamiento similar al de un día domingo.
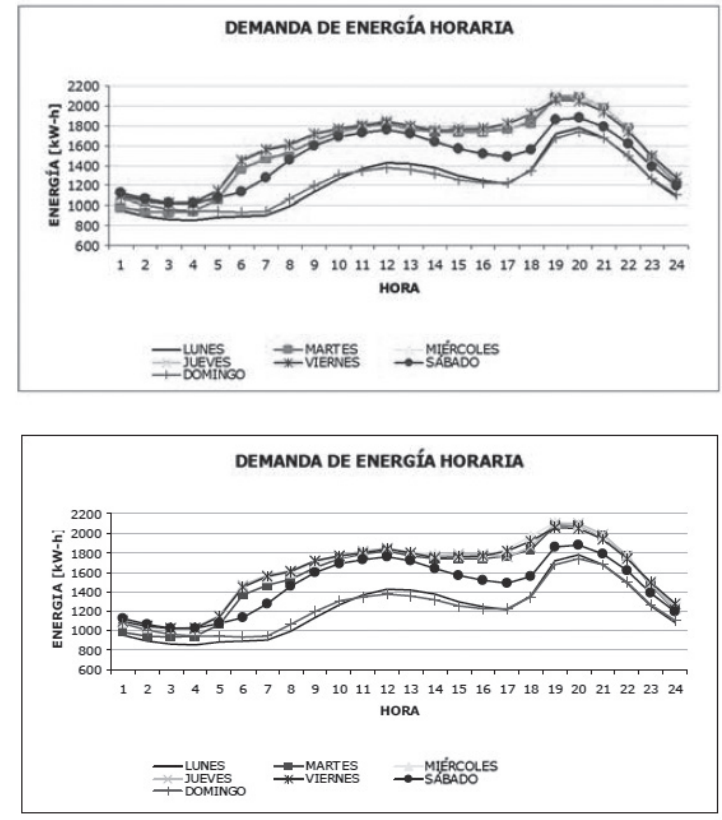

Figura 2. Demanda de energía horaria de una semana típica.

Con base en esta observación, a la tipificación del día se le agregó una variable que determina qué día de la semana presenta un día festivo. En la demanda de energía eléctrica existen otros días especiales a los que se les debe prestar atención ya que su comportamiento presenta ciertos cambios, estos días son el 24 y el 31 de diciembre. Este comportamiento suele ser similar al de un día sábado, como puede apreciarse en la Figura 3. Por esta razón, las anteriores fechas también entran en la tipificación del día. 


\section{con-ciencias}
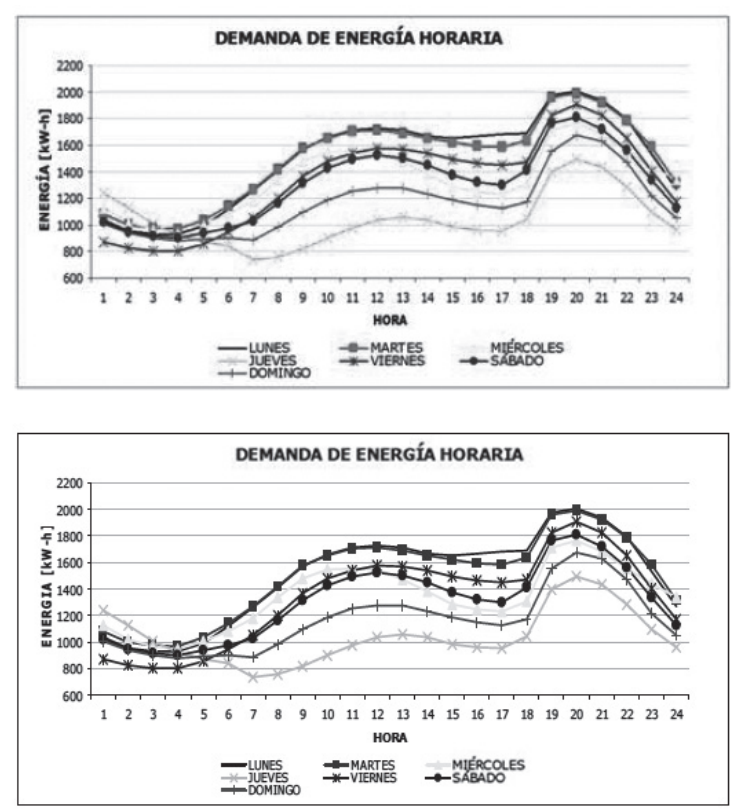

Figura 3. Demanda de energía horaria de una semana con un día 24 de diciembre.
La semana dada a conocer en la Figura 3 es muy especial ya que el día 25 de diciembre es un día festivo, se puede ver su comportamiento a través de la figura. En este caso el día 25 de diciembre es un jueves, la curva tiene una forma muy similar a la de un día sábado y en la misma gráfica para el día 25 de diciembre se observa un comportamiento diferente al de otras fechas, ya que en las tres primeras horas de la mañana la curva de demanda presenta una pronunciada diferencia, este mismo comportamiento ocurre el primero de enero de cada año.

\subsubsection{Días festivos}

En Colombia existen 18 días festivos en un año, este reconocimiento viene de la ley 51 de 1983, más comúnmente conocida como ley Emiliani, en ésta se estipula cuáles son los días de descanso remunerados, los cuales se dan a conocer en la Tabla 2 [8]:

Tabla 2. Días festivos para Colombia.

\begin{tabular}{|c|c|c|c|c|}
\hline FESTIVOS & FECHA & TIPO & CLASE & CELEBRACIÓN \\
\hline 1 & 01-Ene & Religioso & Inamovible & Circuncisión de Cristo \\
\hline 2 & 08-Dic & Religioso & Inamovible & Inmaculada Concepción \\
\hline 3 & 25-Dic & Religioso & Inamovible & Navidad \\
\hline 4 & 01-May & Cívicas & Inamovible & Día del trabajo \\
\hline 5 & 20-Jul & Cívicas & Inamovible & Independencia \\
\hline 6 & 07-Ago & Cívicas & Inamovible & Batalla de Boyacá \\
\hline 7 & 06-Ene & Religioso & Trasladable & Epifanía \\
\hline 8 & 19-Mar & Religioso & Trasladable & San José \\
\hline 9 & 29-Jun & Religioso & Trasladable & San Pedro y San Pablo \\
\hline 10 & 15-Ago & Religioso & Trasladable & Asunción de Ntra. Sra. \\
\hline 11 & 01-Nov & Religioso & Trasladable & Todos los santos \\
\hline 12 & 12-Oct & Cívicas & Trasladable & Día de la raza \\
\hline 13 & $11-\mathrm{Nov}$ & Cívicas & Trasladable & Independencia de Cartagena \\
\hline 14 & $\begin{array}{c}3 \text { días } \\
\text { después de } \\
\text { la pascua }\end{array}$ & Religioso & Trasladable & Jueves santo \\
\hline 15 & $\begin{array}{c}2 \text { días } \\
\text { después de } \\
\text { la pascua }\end{array}$ & Religioso & Trasladable & Viernes santo \\
\hline 16 & $\begin{array}{c}39 \text { días } \\
\text { después de } \\
\text { la pascua }\end{array}$ & Religioso & Trasladable & Ascensión del Señor \\
\hline 17 & $\begin{array}{c}60 \text { días } \\
\text { después de } \\
\text { la pascua }\end{array}$ & Religioso & Trasladable & Corpus Christi \\
\hline 18 & $\begin{array}{l}68 \text { días } \\
\text { después de } \\
\text { la pascua }\end{array}$ & Cívicas & Trasladable & Sagrado Corazón \\
\hline
\end{tabular}




\section{con-ciencias}

Los días festivos se encuentran divididos en dos clases, los días trasladables y los inamovibles; los primeros indican que cuando no caen en un día lunes se trasladarán al lunes siguiente, mientras que los inamovibles son aquellos que se celebran en el día correspondiente (Tabla 2).

De igual manera, los días festivos se encuentran divididos en dos tipos: los cívicos y los religiosos, algunas de las fiestas religiosas están regidas por el calendario eclesiástico, lo cual se puede apreciar en la Tabla 3 [9].

A partir de la anterior información, se obtienen los días festivos de toda la serie y se agrega a estas fechas la tipificación del día respectivo, debido a que, como se dijo anteriormente, estos días presentan comportamientos diferentes en su curva de demanda.

Tabla 3. Fechas de luna llena pascual de los 300 años 1900 a 2199 DC.

\begin{tabular}{|c|c|c|c|c|c|c|c|c|c|c|c|c|c|c|c|c|c|c|c|}
\hline & & & & & & & & & & & & & & & & & & & \\
\hline $\begin{array}{c}\text { Resto } \\
\text { después de } \\
\text { dividir por } \\
19 \text { años }\end{array}$ & 0 & 1 & 2 & 3 & 4 & 5 & 6 & 7 & 8 & 9 & 10 & 11 & 12 & 13 & 14 & 15 & 16 & 17 & 18 \\
\hline $\begin{array}{l}\text { Fecha de } \\
\text { luna llena } \\
\text { pascual }\end{array}$ & 14-abr & 03-abr & 23-mar & 11-abr & 31-mar & 18-abr & $08-a b r$ & 28-may & 16-abr & $05-a b r$ & 25-mar & 13-abr & $02-a b r$ & 22-mar & 10-abr & $30-\operatorname{mar}$ & 17-abr & $07-a b r$ & $27-\mathrm{mar}$ \\
\hline
\end{tabular}

\subsubsection{Semana del mes}

Con el fin de realizar una clasificación más rigurosa, dentro de la tipificación de los días en relación al mes, se creó otra variable que identifica la ubicación del día de la semana dentro del mes. Lo anterior se implementa debido a una pequeña tendencia de aumento detectada en los días que se encuentran en la primera y tercera semana del mes.

\subsubsection{Vacaciones}

Otro tipo de comportamiento particular de demanda se encuentra en lo que llamamos vacaciones, a las que hacen parte unos días de julio, las dos últimas semanas de diciembre y las primeras de enero.

En la Figura 4 se presenta el comportamiento de demanda de las horas $(21,22,23,24)$ para los años 2007 y 2008 , en donde se observa claramente el cambio pronunciado que se da en las vacaciones.

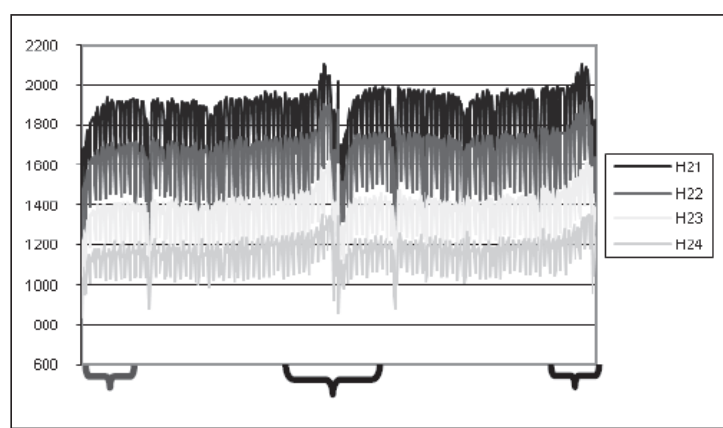

Figura 4. Demanda de energía horaria 2007-2008.

\subsubsection{Semana de puente}

En la Figura 2 se presenta una semana típica con lunes festivo, dentro de la tipificación se vinculó una variable para identificar los días sábados, domingos y martes, antes y después del festivo respectivamente, ya que se encontró que aunque no hay variación de la forma como se observa en la Figura 1, su magnitud varía con respecto a una semana normal. 


\section{con-ciencias}

\subsection{Filtros dinámicos}

A partir de la tipificación descrita en la sección IV-B, se definieron en total ocho variables que generalizan todas las posibles características que se dan dentro de la serie; en la Tabla 4 se da a conocer una breve descripción de cada una.

Tabla 4. Filtros para demanda de energía.

\begin{tabular}{|c|c|c|}
\hline FILTRO & NOMBRE & DESCRIPCIÓN \\
\hline F 1 & AÑO & $>=2003$ \\
\hline F 2 & MES & $1-12$ \\
\hline F 3 & DÍA & $1-31$ \\
\hline F 4 & DÍA DE LA SEMANA & $1-7$ \\
\hline F 5 & TIPO DE FESTIVO & $0-18$ \\
\hline F 6 & DÍAS CON FESTIVOS & $0-19$ \\
\hline F 7 & DÍAS DE VACACIONES & 0 y 20 \\
\hline F 8 & SEMANA DEL MES & $1-5$ \\
\hline
\end{tabular}

Los filtros dinámicos consisten en determinar el filtro que servirá de entrada a los modelos de pronóstico, a partir del dato que establece la fecha a pronosticar. Lo anterior se logra con la ayuda de una aplicación que realiza la tipificación de las fechas de entrada y con éstas se establece el filtro determinado dentro del histórico.

Después de un análisis de los diferentes comportamientos de demanda, que se realizaron con ayuda de gráficas y métodos matemáticos, se desarrollaron alrededor de 30 filtros que en conjunto describen en un $95 \%$ los comportamientos de demanda.

\section{Modelos de pronóstico}

Antes de presentar el modelo desarrollado para este proyecto es necesario aclarar que con base en los análisis realizados en las secciones anteriores, el pronóstico se realiza por hora, es decir, para pronosticar la demanda de un día se tendrán 24 modelos independientes.

Dentro de la identificación de los modelos para realizar el pronóstico de demanda horaria a corto plazo, se estudiaron alrededor de diez métodos diferentes, de los que se escogieron y se adaptaron cuatro por presentar los resultados con menores errores. En las siguientes secciones se realiza una breve descripción de cada uno de éstos.

\subsection{Modelo de promedios móviles}

Una de las principales características de este modelo es que atenúa las fluctuaciones en los datos, enfocando su cálculo a la tendencia de la curva.

Una media móvil utiliza un determinado número de puntos de datos, establecidos por periodo, de los cuales se hace un promedio y se utiliza el valor del promedio como punto de la línea, y a partir de ésta se comienza a formar el comportamiento de la curva, dependiendo de los $\mathrm{k}$ puntos de datos. Por ejemplo, en nuestro caso que el valor del periodo está establecido en dos, el promedio de los dos primeros puntos de datos se utiliza como el primer punto en la línea de tendencia de media móvil. El promedio de los puntos de datos segundo y tercero se utiliza como el segundo punto de la línea de tendencia y así hasta terminar el número de datos de la muestra.

Para este modelo se definió un periodo de dos, lo que significa que todos los datos están directamente relacionados con los últimos dos datos anteriores; como se explicó en la sección 4c, los datos de entrada a cada modelo dependen del filtro, que a su vez está ligado a la tipificación.

La ecuación (2) describe el modelo utilizado para el pronóstico de demanda horaria a corto plazo con el modelo de promedios móviles:

$$
D_{t}=\sum_{i=1}^{n} \frac{d_{t-i}}{n}
$$




\section{con-ciencias}

Donde:

$D_{t}=$ pronóstico de demanda de energía para el periodo $\mathrm{t}$.

$n=$ número de periodos incluidos en el promedio de orden 2 .

$\mathrm{d}_{\mathrm{t}-\mathrm{i}}=$ demanda del periodo $t-i$.

\subsection{Modelo potencial}

El modelo potencial utilizado para el pronóstico de demanda horaria a corto plazo está dado por las expresiones:

$$
\begin{gathered}
y=a \cdot x^{b} \\
\log y=b \cdot \log x+\log a
\end{gathered}
$$

Si $D=\log y$ (eje vertical) y $D=\log x$ (eje horizontal) se tiene que,

$$
D=b \cdot X+\log a
$$

dónde :

$b=$ pendiente de la recta

$\log a=$ coeficiente de posición de la recta.

$D=$ pronóstico de demanda de energía.

$X=$ curva de demanda tipificada.

El modelo potencial es ideal para el tipo de gráficas que tienen un pequeño comportamiento curvo, aproximando a través de rectas. Es pertinente anunciar que este modelo hay que llevarlo a la forma de una ecuación lineal $(y=a x+b)$ con el fin de usar el método de mínimos cuadrados para encontrar su respectiva ecuación.

\subsection{ModeloARMAX (Autoregressive moving average model with exogenous inputs model)}

La notación del modelo está dada por ARMAX ( $p$, $q, b)$ y se refiere a un modelo econométrico con $p$ términos autorregresivos, $q$ términos de media móvil y $b$ términos de entrada exógena. Un modelo ARMAX se compone de un modelo AR (p), un modelo MA (q) y una combinación lineal de los últimos términos de b (entrada exógena). Los modelos autorregresivos se abrevian con la palabra AR y posteriormente se indica el orden del modelo: $\mathrm{AR}(1), \mathrm{AR}(2), \ldots .$. etc. El orden del modelo expresa el número de observaciones retrasadas de la serie temporal analizada que intervienen en la ecuación. La expresión que describe este modelo es:

$Y_{t}=\phi_{0}+\phi_{1} Y_{t-1}+\phi_{2} Y_{t-2}+\ldots+\phi_{t-p} Y_{t-p}+a_{t}$

Los modelos de medias móviles son aquellos que explican el valor de una determinada variable en un periodo $t$, en función de un término independiente y una sucesión de errores correspondientes a períodos precedentes, ponderados convenientemente. Estos modelos se denotan normalmente con las siglas MA, seguidos, como en el caso de los modelos autorregresivos, del orden entre paréntesis. Así, un modelo con $q$ términos de error $\operatorname{MA}(q)$ respondería a la siguiente expresión:

$$
Y_{t}=\mu+a_{t}+\theta_{1} a_{t-1}+\theta 2 a_{t-2}+\ldots+\theta_{q} a_{t-q}
$$




\section{con-ciencias}

Este modelo se estudia en detalle en [10].

Los parámetros de este modelo para el pronóstico de demanda de energía se calculan de manera dinámica a partir del filtro que se seleccione como entrada al modelo, ver sección 4c.

\subsection{Red neuronal}

No existe una definición general de red neuronal artificial, a pesar de que existen algunas aproximaciones del concepto en algunos textos y autores consultados. Una de las definiciones que se identifica más con el modelo realizado en este proyecto es: "Modelos matemáticos desarrollados para emular el cerebro humano" [5]. Las redes neuronales tienen una estructura de cálculo distribuido y son sistemas no lineales, por lo que una interconexión de ellas también será un dispositivo no lineal. Otra propiedad característica de las redes neuronales artificiales (RNA) es que son sistemas tolerantes a fallas, es decir, permiten la falla de algunos de los elementos individuales (neuronas) sin alterar significativamente la respuesta total del sistema, además la red neuronal tiene la capacidad de modificar los parámetros de los que depende su funcionamiento de acuerdo con los cambios que se produzcan en su entorno de trabajo (cambios en las entradas, presencia de ruido, entre otras.), lo que los hace ser un sistema adaptativo ideal para el desarrollo de este proyecto.

Después de realizar diversas pruebas, se desarrolló un modelo de red neuronal multicapa (ver Figura 5) con algoritmo de aprendizaje Backpropagation y función de activación logística.

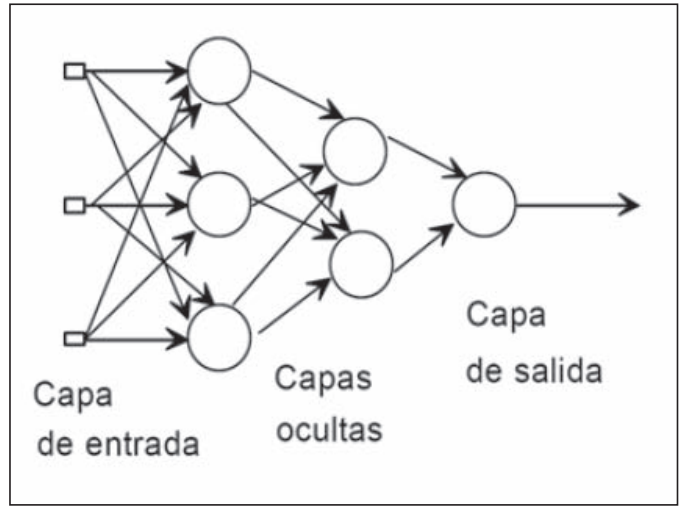

Figura 5. Estructura generalde la red neuronal multicapa.

Este tipo de red neuronal se explica en detalle en [11].

El modelo desarrollado consta de tres neuronas en la capa de entrada, que en nuestro caso representan las tres últimas demandas anteriores al dato a pronosticar, la red está modelada con 2 capas ocultas, la primera con diez neuronas y la segunda con tres, por último una capa de salida con una neurona que representa el dato de demanda pronosticado.

El número de iteraciones se determinó en 200, valor en el que se garantiza el error de $10^{-2}$ en el entrenamiento de la red, el cual es dinámico y se entrena dependiendo del filtro resultado de la tipificación (ver secciones 4B-C, a través de estos valores se realiza una normalización entre $0-1$ de todos los datos que sirven como entrada a la red.

Para más detalles de los modelos desarrollados en el proyecto, el código de la aplicación queda abierto y disponible para su respectiva revisión.

\section{Resultados}

Con el objeto de validar la metodología planteada y establecer los parámetros de los diferentes modelos desarrollados, se realizaron varios ensayos cuyos resultados se presentan en esta parte del artículo. 


\section{con-ciencias}

Se realizaron pruebas de todos los métodos y se comenzó a identificar los modelos finales para cada caso encontrado dentro del análisis de la serie de demanda, donde se validó la estructura final del modelo con una serie de pronósticos que incluyeron 525 datos, de los cuales se obtuvo el comportamiento presentado en la Figura 6.

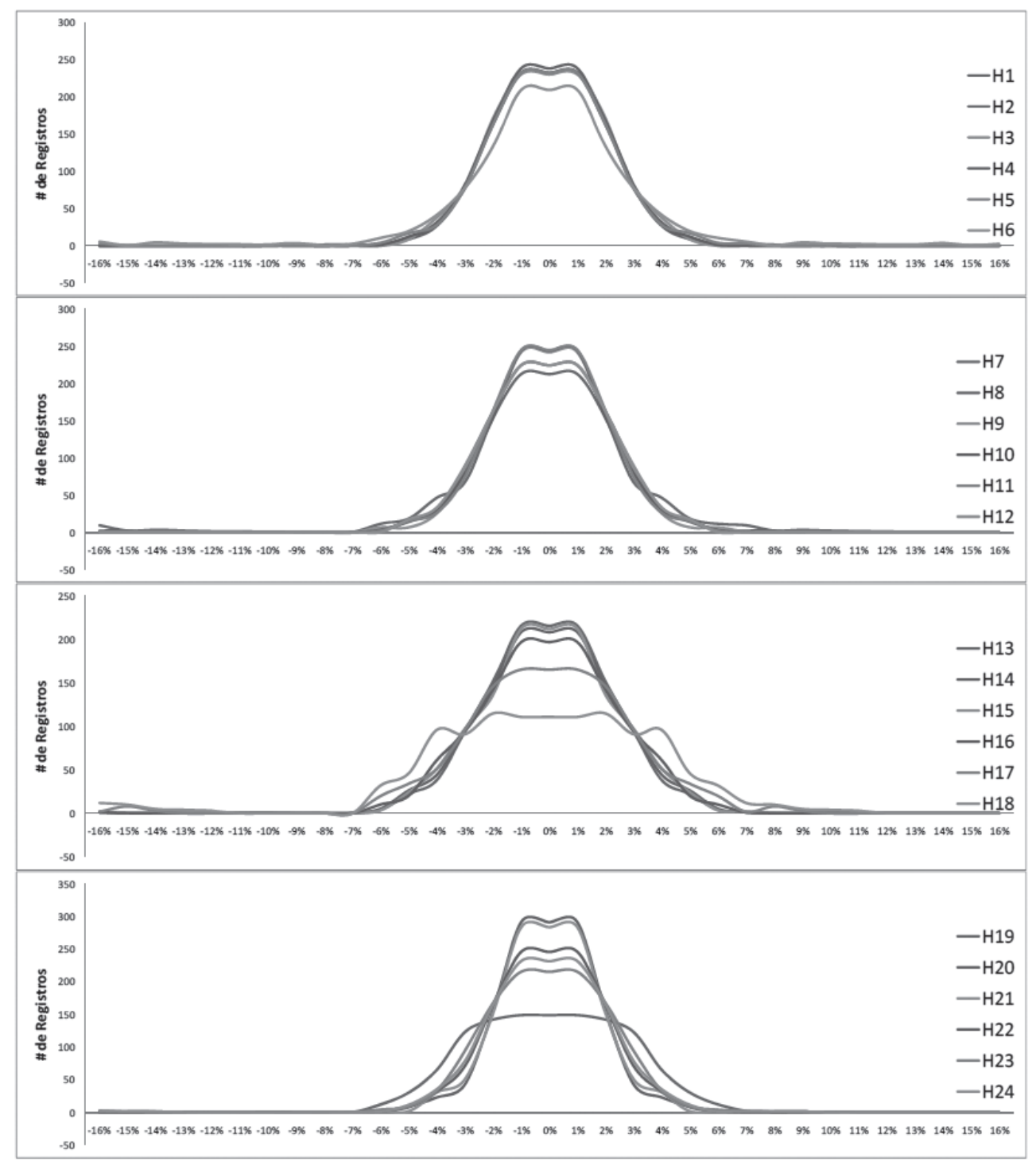

Figura 6. Comportamiento del pronóstico de demanda hora-hora. 


\section{con-ciencias}

Tabla 5. Comportamiento de pronósticos con una muestra de 525 registros.

\begin{tabular}{|c|c|c|c|c|c|c|c|c|c|c|c|c|c|c|c|c|c|c|c|c|c|c|c|c|}
\hline$\%$ & H1 & H2 & H3 & $\mathrm{H} 4$ & H5 & H6 & H7 & H8 & H9 & H10 & H11 & H12 & H13 & H14 & H15 & H16 & H17 & H18 & H19 & $\mathrm{H} 2 \mathrm{O}$ & H21 & H22 & H23 & +24 \\
\hline $16 \%$ & 0 & 0 & 0 & 0 & 3 & 6 & 9 & 2 & 1 & 0 & 0 & 0 & 1 & 1 & 2 & 1 & 2 & 12 & 2 & 1 & 1 & 0 & 1 & 2 \\
\hline $15 \%$ & 0 & 0 & 0 & 0 & 0 & 1 & 2 & 2 & 0 & 0 & 0 & 0 & 0 & 0 & 0 & 0 & 8 & 10 & 1 & 0 & 1 & 0 & 1 & 1 \\
\hline $14 \%$ & 0 & 0 & 0 & 0 & 0 & 5 & 3 & 0 & 0 & 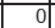 & 0 & 0 & 0 & 0 & 0 & ( & -1 & 5 & 1 & 0 & 0 & 0 & 1 & \\
\hline $13 \%$ & 0 & 0 & 0 & 0 & 0 & 3 & 2 & 0 & 0 & 0 & 0 & 0 & 0 & 0 & 0 & 0 & 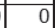 & 4 & 0 & 0 & 0 & 0 & 0 & 0 \\
\hline $12 \%$ & 0 & 0 & 0 & 0 & 0 & 3 & 1 & 0 & 0 & 0 & 0 & 0 & 0 & 0 & 0 & 0 & 0 & 3 & 0 & 0 & 0 & 0 & 0 & U \\
\hline $11 \%$ & 0 & 0 & 0 & 0 & 0 & 2 & 1 & 0 & 0 & 0 & 0 & 0 & 0 & 0 & 0 & 0 & 0 & 0 & 0 & 0 & 0 & 0 & 0 & $\overline{0}$ \\
\hline $10 \%$ & 0 & 0 & 0 & 0 & 0 & 2 & 0 & 0 & 0 & 0 & 0 & $\overline{0}$ & 0 & 7 & 0 & 0 & 0 & 0 & 0 & 7 & 0 & $\overline{0}$ & 0 & \\
\hline $9 \%$ & 1 & 0 & 0 & 0 & 0 & 4 & 0 & 0 & 0 & 4 & $c$ & 4 & 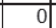 & & 0 & & 0 & 0 & 0 & 0 & 0 & 0 & 0 & \\
\hline $8 \%$ & 2 & 0 & 0 & 0 & 0 & 1 & 0 & 0 & 0 & 0 & 0 & 0 & 0 & 0 & c & 0 & 0 & 0 & 0 & 0 & 0 & 0 & 0 & 0 \\
\hline $7 \%$ & 2 & 0 & 0 & 0 & 0 & 3 & 0 & 0 & 0 & 8 & 0 & 0 & 0 & 0 & 0 & 0 & 0 & 0 & 0 & 0 & 0 & 0 & 0 & 0 \\
\hline $6 \%$ & 2 & 3 & 0 & 1 & 4 & 11 & 11 & 3 & 7 & 3 & 2 & 0 & 4 & 5 & 3 & 10 & 20 & 32 & 12 & 2 & 3 & 3 & 4 & 1 \\
\hline $5 \%$ & 11 & 9 & 9 & 12 & 17 & 20 & 18 & 15 & 7 & 14 & 15 & 17 & 22 & 26 & 24 & 20 & 34 & 46 & 30 & Y & 2 & 8 & 9 & 11 \\
\hline $4 \%$ & 32 & 27 & 38 & 29 & 28 & 42 & 46 & 30 & \begin{tabular}{|l|}
26 \\
\end{tabular} & 30 & 26 & 33 & 38 & 4 & 51 & 61 & 51 & \begin{tabular}{l|l}
96 \\
\end{tabular} & 65 & 23 & 31 & 32 & 33 & 35 \\
\hline $3 \%$ & 80 & 79 & 77 & 82 & 78 & 78 & 67 & 87 & 85 & 79 & 74 & 89 & 94 & 9 & 97 & 94 & 96 & 91 & \begin{tabular}{|c|}
123 \\
\end{tabular} & 3 & 51 & 71 & 94 & 78 \\
\hline $2 \%$ & \begin{tabular}{|l|}
163 \\
\end{tabular} & 168 & \begin{tabular}{|l|}
171 \\
\end{tabular} & 168 & 163 & 134 & 153 & 162 & \begin{tabular}{|l|}
157 \\
\end{tabular} & \begin{tabular}{|c|}
155 \\
\end{tabular} & \begin{tabular}{|l|}
163 \\
\end{tabular} & 162 & \begin{tabular}{|c|}
151 \\
\end{tabular} & 146 & 136 & 142 & 146 & \begin{tabular}{|l|}
115 \\
\end{tabular} & \begin{tabular}{|c|}
142 \\
\end{tabular} & 156 & 152 & 165 & \begin{tabular}{|c|}
167 \\
\end{tabular} & 165 \\
\hline $1 \%$ & 232 & 239 & 230 & 233 & 232 & 210 & 212 & 224 & 242 & \begin{tabular}{|l|}
244 \\
\end{tabular} & 245 & \begin{tabular}{|l|}
224 \\
\end{tabular} & \begin{tabular}{|l|}
215 \\
\end{tabular} & 208 & 212 & \begin{tabular}{l|}
97 \\
\end{tabular} & 165 & 11 & \begin{tabular}{|l|}
149 \\
\end{tabular} & 291 & 284 & 246 & 215 & 231 \\
\hline $0 \%$ & \begin{tabular}{|l|}
232 \\
\end{tabular} & 239 & 230 & 233 & 232 & 210 & 212 & 224 & 242 & 244 & 245 & 224 & 215 & 208 & 212 & \begin{tabular}{|l|}
197 \\
\end{tabular} & 165 & 111 & \begin{tabular}{|c|}
149 \\
\end{tabular} & 291 & 284 & 246 & 215 & \begin{tabular}{|l|l}
231 \\
\end{tabular} \\
\hline$-1 \%$ & \begin{tabular}{|l|}
232 \\
\end{tabular} & 239 & 230 & 233 & 232 & 210 & 212 & 224 & 242 & \begin{tabular}{|l|}
244 \\
\end{tabular} & \begin{tabular}{|l|}
245 \\
\end{tabular} & 224 & 215 & 208 & 212 & \begin{tabular}{|l|}
197 \\
\end{tabular} & 165 & 111 & \begin{tabular}{|l|}
149 \\
\end{tabular} & 291 & 284 & 246 & \begin{tabular}{|l|}
215 \\
\end{tabular} & 231 \\
\hline$-2 \%$ & \begin{tabular}{|l|}
163 \\
\end{tabular} & 168 & \begin{tabular}{|l|}
171 \\
\end{tabular} & 168 & 163 & 134 & 153 & 162 & \begin{tabular}{|c|}
157 \\
\end{tabular} & \begin{tabular}{|c|}
155 \\
\end{tabular} & \begin{tabular}{|l|}
163 \\
\end{tabular} & \begin{tabular}{|c|}
162 \\
\end{tabular} & \begin{tabular}{|l|}
151 \\
\end{tabular} & 146 & 136 & \begin{tabular}{|l|}
142 \\
\end{tabular} & 146 & 115 & \begin{tabular}{|l|l|}
142 \\
\end{tabular} & 156 & 152 & 165 & \begin{tabular}{|c|}
167 \\
\end{tabular} & 165 \\
\hline$-3 \%$ & 80 & 79 & 77 & 82 & 78 & 78 & 67 & 87 & 85 & 79 & 74 & 89 & 94 & 95 & 97 & 94 & 96 & 91 & \begin{tabular}{|c|}
123 \\
\end{tabular} & 43 & 51 & 71 & 94 & 78 \\
\hline$-4 \%$ & 32 & 27 & 38 & 29 & 28 & 42 & 46 & 30 & 26 & 30 & 26 & 33 & 38 & 44 & 51 & 61 & 51 & 96 & 65 & 23 & 31 & 32 & 33 & 35 \\
\hline$-5 \%$ & 11 & 9 & 9 & 12 & 17 & 20 & 18 & 15 & 7 & 14 & 15 & 17 & 22 & 26 & 24 & 20 & 34 & $\begin{array}{ll}46 \\
\end{array}$ & 30 & 9 & 2 & 8 & 9 & 11 \\
\hline$-6 \%$ & 2 & 3 & 0 & 1 & 4 & 11 & 11 & 3 & 7 & 3 & 2 & 0 & 4 & 5 & 3 & 10 & 20 & 32 & \begin{tabular}{l|}
12 \\
\end{tabular} & 2 & 3 & 3 & 4 & 1 \\
\hline$-7 \%$ & 2 & 0 & 0 & $\overline{0}$ & 3 & $\begin{array}{ll}6 \\
\end{array}$ & 9 & 2 & 1 & 0 & 0 & 0 & 1 & 1 & 2 & 1 & 2 & 12 & 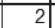 & 1 & 1 & 0 & 4 & 2 \\
\hline$-8 \%$ & 2 & 0 & 0 & 0 & 0 & 1 & 2 & 2 & 0 & 0 & 0 & 0 & 0 & 0 & 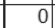 & 0 & 8 & 10 & 1 & 0 & 1 & 0 & 1 & 1 \\
\hline$-9 \%$ & 1 & 0 & 0 & 0 & 0 & 5 & 3 & 0 & 0 & 0 & 0 & 0 & 0 & 0 & 0 & & 3 & 5 & 1 & 0 & 0 & $\overline{0}$ & 1 & 1 \\
\hline$-10 \%$ & 0 & 0 & 0 & 0 & 0 & 3 & 2 & 0 & 0 & 4 & 0 & 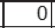 & 0 & 0 & 0 & 0 & 0 & 4 & 0 & 0 & 0 & 0 & 0 & 0 \\
\hline-11 & 0 & 0 & 0 & 0 & 0 & 3 & 1 & 0 & 0 & 0 & & 0 & 0 & & ( & & & 3 & & & 0 & & 0 & 0 \\
\hline$-12 \%$ & 0 & 0 & 0 & 0 & 0 & 2 & 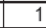 & $\overline{0}$ & $\overline{0}$ & 0 & & 0 & 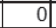 & & & & & 0 & & & 0 & & & 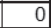 \\
\hline$-13 \%$ & 0 & 0 & 0 & 0 & 0 & 2 & 0 & 0 & 0 & 0 & 0 & 0 & 0 & 0 & 0 & 0 & 0 & 0 & 0 & 0 & 0 & 0 & 0 & 0 \\
\hline$-14 \%$ & 0 & 0 & 0 & 0 & 0 & 4 & 0 & 0 & 0 & 0 & 0 & 0 & 0 & 0 & 0 & 0 & 0 & 0 & 0 & 0 & 0 & 7 & 0 & 0 \\
\hline$-15 \%$ & 0 & 0 & 0 & 0 & 0 & 1 & 0 & 0 & 0 & 0 & 0 & 0 & 0 & 0 & 0 & 0 & 0 & 0 & 0 & 0 & 0 & 0 & 0 & 0 \\
\hline$-16 \%$ & 0 & 0 & 0 & 0 & 0 & 3 & 0 & 0 & 0 & 0 & 0 & 0 & 0 & 0 & 0 & 0 & 0 & 0 & 0 & 0 & 0 & 0 & 0 & 0 \\
\hline
\end{tabular}

La Figura 6 muestra el comportamiento del pronóstico de cada hora, este resultado se calculó al comparar el pronóstico con el histórico de demanda, aproximadamente un $75 \%$ de los días pronosticados se encuentran con un error menor al $2 \%$, el $87 \%$ dentro del $3 \%$, el $95 \%$ dentro del $4 \%$ y el $98 \%$ dentro de un error del $5 \%$. El porcentaje hora-hora de los datos dentro de cada rango se presenta en la Tabla. 6.

Tabla 6. Comportamiento de pronóstico hora-hora en porcentaje.

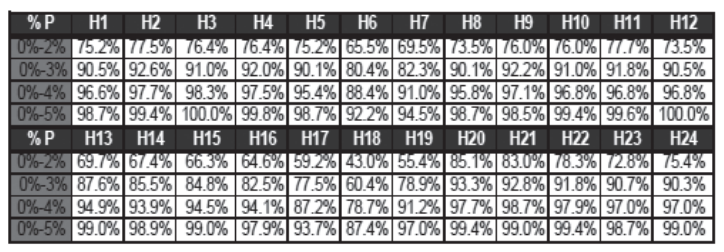

este comportamiento y se evidencia el rango de variación del pronóstico, mostrando esta curva un poco más achatada a las demás, aumentando así la dispersión del pronóstico de estas horas. Para los otros casos, tenemos que el comportamiento de los pronósticos hora-hora presenta un valor más estable y aproximadamente el $90 \%$ de la estimación estuvo dentro de un margen de error del $\pm 3 \%$.

Otra de las pruebas fue calcular el pronóstico de demanda de los meses de mayo y junio del presente año y observar el comportamiento durante cada mes, este análisis se realiza con base a que XM y la CREG validan la información con el promedio de los errores de todo el mes.

Como se puede observar, los errores con un porcentaje mayor se presentan en las horas 18 y 23 , y presentan una gran aleatoriedad en la demanda durante estos periodos; en la Figura 6 se observa 


\section{con-ciencias}

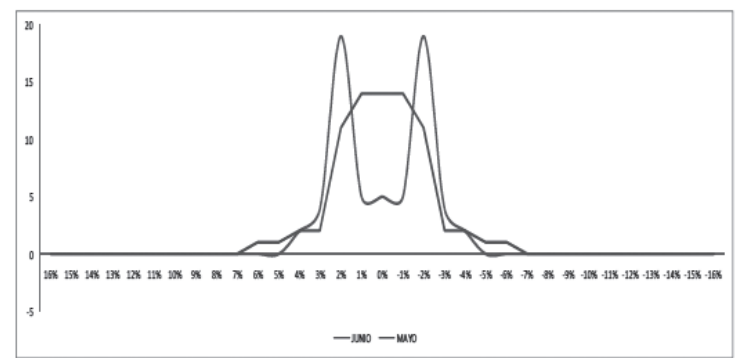

Figura 7. Comportamiento del pronóstico de demanda mayo-junio 2009.

El error promedio para el mes de mayo y junio es de $1.65 \%$ y $1.56 \%$ respectivamente, en la Figura 7 se presenta el comportamiento de los pronósticos y se observa que para junio la estimación está dentro de un margen de error del $\pm 2.5 \%$ y para mayo entre el $\pm 2 \%$. Sin embargo, aunque el rango de error es mayor para el mes de junio, presenta un mejor valor durante el mes, como se muestra en la distribución de los datos en la Figura 7.

Los resultados obtenidos en las pruebas de validación fueron acordes dentro del alcance del proyecto y comparándolos con los pronósticos actuales, la aplicación presenta un mejor desempeño.

\subsection{Prototipo}

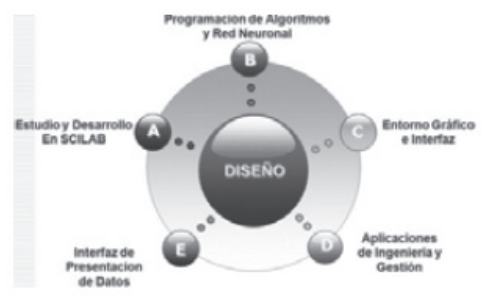

Figura 8. Proceso general para el diseño del soporte lógico. Fuente: elaboración propia.

El diseño del prototipo para el soporte lógico se desarrolló bajo la metodología mostrada en la Figura 8. Dentro del desarrollo del software se programaron varias aplicaciones que componen en conjunto la entrada y la salida de información, de acuerdo como se utiliza en Codensa S.A. (formato Excel) y como se reporta a la CREG y al CND (formato archivo plano.txt). Adicionalmente, una parte de la interfaz está orientada a visualizar los datos pronosticados, con el fin de que el usuario tenga una retroalimentación gráfica del resultado del pronóstico que se obtuvo.

Por otro lado, se realizó un estudio acerca de la manera en que se debe desarrollar una aplicación informática y de la forma de implementar una interfaz de usuario, esta parte se estudia en [12].

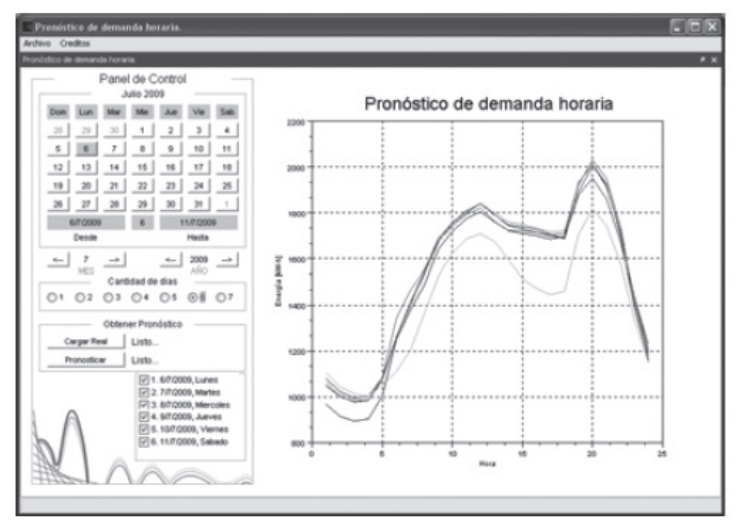

Figura 9. Interfaz gráfica"Pronóstico de demanda horaria". Fuente: Elaboración propia.

En la Figura 9 se presenta la interfaz gráfica desarrollada en este proyecto, la cual se divide en tresáreas generales, la primera consiste en asignar las variables de entrada como el ingreso de las fechas que se desean pronosticar, la segunda está definida por los botones que ejecutan las aplicaciones de importación de datos de demanda histórica y otro que sirve para el cálculo de pronóstico, y la tercera representa la gráfica donde se muestra el comportamiento de las curvas pronosticadas.

La aplicación está desarrollada bajo una plataforma de java y corre bajo Scilab 5.1, software libre de código abierto. Los modelos se desarrollaron bajo 


\section{con-ciencias}

funciones, lo cual optimizó el tiempo de ejecución de la aplicación, además se utilizaron varios complementos que están disponibles en [13].

\section{Conclusiones}

Tanto el modelo como la aplicación desarrollada en el proyecto presentan un mejor desempeño a la aplicación actual de Codensa S.A., mejorando en promedio el error del pronóstico en un $2 \%$.

Las horas 18 y 23 presentan un comportamiento levemente aleatorio que dificultó el desarrollo del modelo que describe su comportamiento, obteniendo el mejor resultado con el modelo que se implementó en la aplicación.

Dentro del análisis y desarrollo del proyecto se encontró que es necesario utilizar diferentes modelos para describir todos los casos posibles de demanda. No se recomienda utilizar un solo modelo debido al comportamiento que presenta la demanda.

El desarrollo del soporte lógico bajo la plataforma de un software matemático como Scilab trae múltiples beneficios a futuro, más la posibilidad de seguir mejorando el modelo y hacer gestión con los datos. Toda la aplicación está realizada bajo software libre, lo que fomenta el desarrollo de la misión de la Universidad Distrital.

\section{Agradecimientos}

Los autores reconocen la colaboración de los profesionales Libardo Villamizar y José María Salas de Codensa S.A.

\section{Referencias bibliográficas}

[1] CREG, Resolución CREG 097 de 2008. "Por la cual se aprueban los principios generales y la metodología para el establecimiento de los cargos por uso de los Sistemas de Transmisión Regional y Distribución Local”. [En línea]. Disponible en:http://www. creg.gov.co/html/i_portals/index.php?p_ origin $=$ internal\&p_name $=$ content $\& p_{-}$ id=MI-75\&p_options=

[2] A.L.Valencia, C.A. Lozano, C.A. Moreno "Modelo de promedios móviles para el pronóstico horario de potencia y energía eléctrica",artículo de Maestría, Universidad Autónoma de Occidente, Cali, Colombia, pp.2-11, diciembre 2007.

[3] J. Murillo, A. Trejos y P. Carvajal, "Estudio del pronóstico de la demanda de energía eléctrica, utilizando modelos de series de tiempo", artículode Maestría, Universidad Tecnológica de Pereira,pp.3-5, diciembre2004.

[4] UPME, "Proyecciones por Unidad de Control de Pronostico (UCP) energía y potencia", Revisión, 2007.

[5] C. T. Chen, W. D. Chang, "A Feedforward Neural Network with Function Shape Autotuning",Neural Networks, vol. 9, no. 4, pp. 627-641, 1996.

[6] J. Moreno, "Control de la Demanda de Energía Eléctrica", Reportaje Red Eléctrica de España, pp. 20-24, agosto 2006.

[7] E.E. Ramírez, "Pronóstico de demanda de energía eléctrica utilizando regresión lineal", 


\section{con-ciencias}

Tesis de pregrado, Instituto Politécnico Nacional, 2008.

[8] Ley 51 de 1983 (Ley Emiliani) [en línea]. Disponible en: http://www.rebayona.com/ outlook/emiliani.htm.

[9] Computus, Gregorian Calendar [en línea]. Disponible en: http://en.wikipedia.org/wiki/ Computus.

[10] G, Damodar, Econometría.McGRAW-HILL, edición 4, pp.765-840, 26, septiembre 2003.
[11] E. Soria, J. Martin, Procesado Inteligente de la Información, Departamento de Ingeniería Electrónica:Universidad de Valencia, pp. 2143, 2006.

[12] R. Pressman,Ingeniería Del Software Un Enfoque Práctico,edición 5, pp. 343-521, abril 2006.

[13] Scilab.org [en línea]. Disponible en: http:// www.scilab.org/contrib/index_contrib. php?page $=$ download.php 